\title{
Criminologie
}

\section{La gestion de cas en libération conditionnelle}

\section{Carl Eugène, Marie Sarrasin et Louise Thireau}

Volume 14, numéro 2, 1981

Libération conditionnelle : évolution et application (1899-1981)

URI : https://id.erudit.org/iderudit/017138ar

DOI : https://doi.org/10.7202/017138ar

Aller au sommaire du numéro

Éditeur(s)

Les Presses de l'Université de Montréal

ISSN

0316-0041 (imprimé)

1492-1367 (numérique)

Découvrir la revue

Citer cet article

Eugène, C., Sarrasin, M. \& Thireau, L. (1981). La gestion de cas en libération conditionnelle. Criminologie, 14(2), 25-40. https://doi.org/10.7202/017138ar d'utilisation que vous pouvez consulter en ligne.

https://apropos.erudit.org/fr/usagers/politique-dutilisation/ 


\section{LA GESTION DE CAS EN LIBÉRATION CONDITIONNELLE \\ Carl Eugène \\ Marie Sarrasin \\ Louise Thireau}

La création de la Commission nationale des libérations conditionnelles (C.N.L.C.) a été à l'origine de la création des postes, plus obscurs, d'analystes pour la Commission. Par la suite, lorsque le Service national des libérations conditionnelles (S.N.L.C.) fut mis sur pied, c'est l'agent des libérations conditionnelles qui fut chargé de la préparation des cas sur lesquels la C.N.L.C. statuait en octroyant ou non une libération.

En 1977, le S.N.L.C. est intégré au Service correctionnel canadien (S.C.C.). Cette intégration est à la base des réorganisations administratives qui aboutissent, en janvier 1981, à l'implantation d'une "nouvelle gestion de cas ». Le travail de l'agent de libérations conditionnelles chargé de la préparation de cas se modifie alors sensiblement.

Il nous a paru important, pour bien faire comprendre au lecteur les changements survenus au niveau de la préparation de cas, de décrire, dans un premier temps, le rôle «traditionnel 》 de l'agent de libération et, dans un deuxième temps, les tâches qui lui sont maintenant dévolues selon ce qu'on appelle couramment, la «nouvelle gestion de cas».

Ces deux premières sections nous permettront, dans une troisième partie, de soulever quelques-unes des questions que le professionnel se pose.

Nous nous sommes aussi penchés sur les relations assez étroites qui existent entre la nouvelle gestion de cas implantée par le S.C.C. et l'orientation américaine de la sentence déterminée.

Finalement, le professionnel qui s'occupe de préparation de cas est-il une espèce en voie de disparition ? Nous ne désirons pas donner une réponse à cette question mais plutôt apporter des éléments de réflexion sur notre devenir.

1. L'ÉTUde de CAS ET LE RÔLE DE L'AGENT DES Libérations CONDITIONNELLES

De par ses origines, l'agent de libérations conditionnelles était perçu tant par les institutions que par ses propres membres comme celui qui devait réunir l'ensemble des informations, permettant ultérieurement à la C.N.L.C. de se prononcer sur l'opportunité d'octroyer ou non une libération conditionnelle. 
C'est en ce sens qu'il a longtemps été considéré comme un "conseiller spécial » de la C.N.L.C., à qui il soumettait sa recommandation.

Nous tenterons ici de présenter le travail de l'agent de libérations conditionnelles et de définir le rôle qui lui était attribué et qu'il tentera de préserver à l'intérieur des structures nouvelles.

La préparation de cas

Essentiellement, le travail de l'agent de libérations conditionnelles consiste à produire un rapport qui constitue l'étude et la préparation du cas. Ce document est une évaluation, synthèse ayant pour but de déterminer l'adaptabilité et la dangerosité du détenu par rapport à une éventuelle réinsertion sociale. Elle vise aussi à déterminer les facteurs qui influencent ces deux dimensions fondamentales.

\section{Rôle de l'agent de libérations conditionnelles}

L'agent de libérations conditionnelles doit fournir l'évaluation la plus objective possible. Pour ce faire, il recourt à plusieurs sources d'information, documents et personnes qui le renseignent sur l'individu tel que perçu à l'intérieur des murs et aussi à l'extérieur du pénitencier.

Il peut s'agir d'évaluations d'experts (psychiatres, psychologues), de l'opinion d'agents de probation, de policiers, d'exemployeurs, de parents, etc.

Il appartient également à l'agent d'effectuer une entrevue avec le détenu afin d'établir le contact personnel essentiel à toute évaluation-diagnostic.

\section{Les critères d'évaluation et les sources d'information}

L'article 10 (1) de la Loi des libérations conditionnelles établit trois critères fondamentaux sur lesquels la Commission appuie ses décisions. Le détenu doit avoir tiré le plus grand avantage possible de l'emprisonnement; l'octroi de la libération conditionnelle doit faciliter le redressement et la réhabilitation du détenu et sa mise en liberté ne doit pas constituer un risque indû pour la société.

L'agent doit donc relever les éléments qui lui permettront de juger de la capacité de l'individu à s'intégrer à la société, d'évaluer les risques encourus et, surtout, il doit pouvoir identifier les indices de changement de l'individu.

L'histoire sociale et familiale du sujet, la précocité de l'émergence de la délinquance, la nature de l'acting-out, les fac- 
teurs à la base du passage à l'acte, les caractéristiques de la personnalité du sujet sont, dans un premier temps, des indices du degré de violence potentielle du délinquant.

Le comportement institutionnel, les efforts fournis par le sujet en fonction de ses capacités, ses problèmes disciplinaires rencontrés sont, pour leur part, des indices de la capacité d'adaptation de l'individu face à des cadres et des figures d'autorité.

La qualité et la disponibilité des ressources extérieures le type de relation entre l'individu et ses personnes ressources donnent, dans un troisième temps, des indices quant à la facilité de réinsertion sociale du sujet.

Tous ces indices qui, à première vue, paraissent statiques sont cependant analysés dans une perspective évolutive. C'est en effet, en fonction du «changement » observé chez un individu que l'agent en arrivera à formuler un diagnostic et un pronostic.

Le diagnostic et le pronostic

Le travail diagnostic consiste à synthétiser, analyser, relier et interpréter les informations recueillies afin de déterminer l'état actuel de la délinquance et de l'individu en fonction des changements survenus chez lui.

Quant au pronostic, il consiste en une analyse de la probabilité d'une réinsertion sociale réussie et de la nature d'une éventuelle récidive.

Le diagnostic et le pronostic ne seront valables que dans la mesure où le travail d'évaluation visera à connaître la qualité de l'évolution. Aussi, le conformisme d'un fraudeur ne peut être interprété comme une garantie de changement et de non-récidive. C'est justement dans cette évaluation de la qualité de la motivation, du changement, etc., que l'étude de cas prend sa valeur.

La recherche de ressources

Une partie importante du travail de l'agent des libérations conditionnelles consiste à identifier, évaluer et/ou à rechercher les ressources qui sauront influencer et souvent déterminer l'adaptation sociale.

En effet, le manque de ressources, particulièrement dans les cas spéciaux (débiles, malades mentaux, individus démunis socialement), est un des problèmes auquel est confronté l'agent au moment de la formulation de sa recommandation.

L'agent de libérations conditionnelles, dans son rôle traditionnel, s'intéressait donc autant au passé du sujet, à son vécu 
institutionnel qu'à sa future réinsertion sociale et finalement aux ressources extérieures qui prendront, plus ou moins, la relève lorsque le sujet sera libéré.

Il nous apparaît important, maintenant, de nous demander si, d'une part, la préparation de cas est utile et nécessaire et si, d'autre part, l'agent de libérations a un rôle vraiment essentiel à jouer dans cette préparation?

La réussite de la préparation de cas

Depuis quelques années, un mouvement se dessine dans le domaine de la délinquance qui remet en question la capacité pour les spécialistes des sciences humaines de modifier le comportement délinquant et, surtout, de le prédire et de le prévenir. Aux yeux des administrateurs, l'agent de libérations conditionnelles n'est pas rentable.

La question peut être considérée sous deux angles. Premièrement, la présence d'une personne de l'extérieur est-elle pertinente? Deuxièmement, le travail d'évaluation en vaut-il la peine?

L'importance de l'agent de libérations conditionnelles

La première version de l'implantation de gestion de cas à l'intérieur du Service correctionnel canadien impliquait l'intégration physique des agents de libérations conditionnelles affectés à la préparation de cas à l'intérieur du pénitencier. Ces derniers ont immédiatement souligné que les évaluations perdraient alors de leur objectivité.

Il est certain que les institutions sont dotées d'un personnel qui est en mesure d'effectuer l'évaluation du détenu. Cependant, le fait que ces personnes soient régulièrement en contact direct avec celui-ci et ce, constamment dans le contexte de l'institution, représente une source de biais importants par rapport à une évaluation objective et globale du sujet.

Le piège qui guette le personnel institutionnel est de minimiser le passé du détenu pour accorder une signification trop grande au comportement institutionnel. Une évaluation de l'extérieur a davantage de chance d'être plus «neutre » et plus complète. L'étude de cas est-elle vraiment utile?

Un ordinateur ne pourrait-il pas effectuer plus efficacement le diagnostic et le pronostic de la délinquance? C'est sûrement la question que se posent plusieurs administrateurs.

Il semble peu probable qu'on puisse jamais prouver l'utilité de la préparation de cas mais il existe des facteurs qu'une machine 
ne saurait évaluer. Qu'il suffise de mentionner la saturation criminelle, la qualité des ressources extérieures, la sincérité de l'individu, la qualité de son évolution, etc. Ces éléments sont pourtant d'une grande importance dans l'évaluation du détenu et de ses possibilités de réinsertion sociale.

Il apparaît très hasardeux de fonder la libération conditionnelle sur des critères uniquement concrets et mesurables, car des injustices encore plus grandes que celles observées dans le système actuel risqueraient de se produire et l'individu serait alors déshumanisé. En effet, il perdrait l'élément de base de son être, soit sa capacité d'évolution, donc de changement.

En conclusion, il nous apparaît en somme que l'agent de libérations conditionnelles a un rôle important à jouer, par l'intermédiaire de son évaluation, lorsque vient le moment pour la C.N.L.C. d'octroyer ou non une libération à un individu. C'est toutefois là, si l'on peut dire, son rôle traditionnel. Avec la mise en application de la nouvelle gestion de cas, quel sera son nouveau rôle? C'est ce que nous désirons maintenant aborder.

2. LA NOUVELLE GESTION DE CAS : PRINCIPES ET MODE D'APPLICATION

But de la nouvelle gestion de cas - principes de base sous-jacents

La nouvelle gestion de cas est fondamentalement une réorganisation administrative de ce qu'on appelle aujourd'hui le Service correctionnel canadien (S.C.C.).

Le S.C.C., en effet, a été créé à partir de l'intégration (ou fusion) de deux services originellement distincts, soient le Service canadien des pénitenciers (S.C.P.) et le S.N.L.C.

Cette réorganisation administrative, qui dans les faits n'est que l'absorption pure et simple du S.N.L.C. par le S.C.P., repose théoriquement sur les principes suivants :

a) Impliquer davantage le délinquant dans la gestion de sa peine et dans le processus de sa réinsertion sociale, et

b) Assurer une certaine cohérence dans l'ensemble des programmes offerts par le S.C.C., en mettant l'emphase notamment sur la continuité.

Les éléments de la nouvelle gestion de cas

La nouvelle gestion de cas comprend une nouvelle nomenclature des titres de poste, une nouvelle définition des tâches des fonctionnaires, l'impression de nouveaux formulaires, la revision 
vocationnelle des différentes institutions en fonction de leur degré de sécurité et des programmes offerts et enfin l'établissement d'un nouveau type de rapport entre les délinquants et les employés du service.

A. L'équipe de gestion de cas (E.G.C.)

Dans la réorganisation de la division du travail, la notion d'équipe est celle qui frappe le plus et qui semble avoir le plus d'impact, en particulier sur les ex-agents de libérations conditionnelles assignés dans les bureaux de préparation de cas (Laval, St-Jérôme et Granby pour le Québec).

Jadis, relativement indépendants l'un de l'autre, aussi bien par leurs titres que par leurs rôles, l'ex-agent de libérations conditionnelles et l'ex-agent de classement deviennent, tous les deux, membres d'une équipe de gestion de cas à laquelle incombe désormais la responsabilité, à la fois, de la préparation de cas proprement dite et de l'étude de toute autre action à prendre dans le cours d'exécution d'une sentence (absences temporaires, transferts, etc.).

\section{Composition}

Outre ces deux fonctionnaires, respectivement désignés comme étant l'agent de gestion de cas communautaire (A.G.C.C.), et l'agent de gestion de cas d'établissement (A.G.C.E.), l'équipe de gestion de cas comprend l'agent d'unités résidentielles (A.U.R.), et occasionnellement des membres ad hoc dont la présence est susceptible d'apporter une contribution significative dans l'étude du cas. Ces membres ad hoc peuvent être psychologue, aumônier, agent de sécurité, etc. Jusqu'à un certain point, le délinquant, luimême, peut être considéré comme membre de l'E.G.C., étant donné le rôle actif qu'il est appelé à jouer dans l'élaboration des programmes le concernant.

\section{Le rôle des membres constituants}

L'équipe de gestion de cas s'occupe d'un certain nombre de détenus dont elle suit l'évolution et trace les grandes lignes du processus de réinsertion sociale. Elle se réunit au besoin pour discussion et action et est présidée tantôt par l'A.G.C.C., tantôt par l'A.G.C.E.

C'est l'A.G.C.C. qui est chargé de rédiger les rapports en ce qui concerne les projets orientés vers la collectivité : L.C."

* L.C. : libération conditionnelle. 
S.O.**, L.C. de jour, l'A.G.C.E. rédige les autres rapports, transferts, absences temporaires, etc.

L'A.U.R. et les membres ad hoc informent et éclairent l'E.G.C. sur l'état et l'évolution du cas (comportement, problèmes psychologiques, etc.).

B. Le plan de séjour

L'outil de base dans la nouvelle gestion de cas c'est le plan de programme individualisé (P.P.I.) ou plan de séjour.

Le P.P.I. comprend essentiellement deux parties :

a) Le profil d'analyse des besoins (P.A.B.) qui établit l'ensemble des besoins du sujet (éducation, formation technique, traitement, etc.).

b) Le régistre du plan de programme individualisé (R.P.P.I.) qui trace le programme d'activités susceptibles de répondre aux besoins identifiés dans le P.A.B.

Le plan de séjour est revisé périodiquement en fonction du rythme d'évolution du détenu et des facilités offertes par l'institution.

\section{But du plan de séjour}

Il est à souligner que le plan de séjour, bien qu'impliquant activement le délinquant qui a participé à son élaboration, ne se définit pas comme étant un contrat entre celui-ci et le S.C.C., mais plus simplement un outil de travail. Il sert à assurer une certaine continuité d'action et une certaine cohérence dans la gestion de la sentence en établissant un programme qui couvre aussi bien la période d'incarcération que celle où le délinquant se trouve sous surveillance (L.C. ou S.O.).

\section{Les étapes importantes du P.P.I.}

Comme nous l'avons dit plus haut, le plan de séjour est mis à jour périodiquement. Il l'est, d'abord, par le biais du rapport trimestriel sur l'évolution du cas (R.T.E.C.) ou, comme son nom l'indique, on relève à tous les trois mois les progrès réalisés, ou non, par le délinquant et où on réajuste le programme tracé dans son cas. Il l'est également dans des étapes spécifiques de la sentence, notamment le début d'un programme d'absences temporaires, un transfert d'institution, une demande de libération conditionnelle, etc. $\grave{A}$ chacune de ces étapes, l'E.G.C. produit un rapport récapitulatif sur l'évolution du cas (R.R.E.C.) qui fait le point et traite de la pertinence de l'action considérée.

** S.O. : surveillance obligatoire. 
Nous le voyons, la nouvelle gestion de cas apporte des modifications très importantes au rôle de l'agent en charge de leur préparation. Comment le professionnel qui doit s'y adapter perçoit-il et comment vit-il cette adaptation?

3. RÉFLEXIONS SUR LA MISE EN APPLICATION DE LA NOUVELLE GESTION DE CAS ET SUR LE RÔLE DE L'A.G.C.C.

Dans les deux sections précédentes nous avons résumé le rôle de l'agent de libération conditionnelle avant janvier 1981 et celui qui lui est dévolu depuis l'arrivée de la nouvelle année. Didactiquement nous pourrions dire que l'agent de libération est devenu, comme son nouveau titre de poste l'indique, un agent de gestion de cas.

Comment le professionnel vit-il ce changement? Quel est l'impact de cette nouvelle gestion de cas sur son travail? Quelle est sa marge de manœuvre? Voilà autant de questions sur lesquelles il lui faut réfléchir pour se redéfinir. C'est cette réflexion que nous voulons amorcer dans cette troisième section.

L'impact de la nouvelle gestion de cas sur le rôle d'évaluation de l'agent de libération conditionnelle (A.G.C.C.)

Larousse définit la «gestion» comme suit : «n.f. Action de gérer; administration ». Quant au gestionnaire, Larousse le décrit ainsi : "Gradé charger d'administrer un établissement, un hôpital militaire». Par extension, donc, l'agent de gestion de cas communautaire, qui ouvre au niveau de la préparation de cas, est chargé de participer à la gestion d'une sentence imposée, par un tribunal, à un individu donné.

Dans la procédure mise en cuvre au niveau de la gestion de cas un des éléments qui, à première vue, paraît le plus nouveau est certes l'idée de l'équipe de gestion de cas. Cela signifie que l'évaluation d'un cas est faite, non par un ou des individus pris séparément (agent de classement, agent de libération) mais par l'ensemble de l'E.G.C. Il y a donc, corollairement, l'idée d'en arriver à un consensus au niveau de la recommandation qui sera faite à la C.N.L.C. pour un sujet.

Cette notion de concertation entre l'A.G.C.C. et l'A.G.C.E. qui paraît nouvelle est, dans les faits, beaucoup plus ancienne. En effet, quoique non formelle, une discussion avait presque toujours lieu entre le préposé au classement et l'agent de libération avant même l'instauration de la nouvelle gestion de cas. 
Là où il y a un réel changement, c'est surtout au niveau de la rédaction de l'évaluation elle-même, puisque l'A.G.C.C. ne parle plus en son nom mais en celui de l'E.G.C. De plus, au niveau du contenu même du rapport, l'accent est surtout mis sur le comportement du sujet en institution.

En voulant éviter dans un dossier, les répétitions et la duplication de tâches, ce qui en soit est logique et souhaitable, c'est principalement au niveau de l'étude faite en vue d'une libération que l'évaluation d'un sujet semble devoir perdre en qualité.

En effet, l'évaluation que faisait anciennement l'agent de libération, parce que non centrée principalement sur l'attitude du sujet au pénitencier mais aussi sur les divers aspects de sa personnalité, sur son cheminement criminel, sur les ressources extérieures fournissait un point de référence englobant l'ensemble des renseignements fournis par le dossier, de même qu'un diagnostic et un pronostic plus élaborés.

Un des éléments qui permet d'expliquer l'importance encore plus grande accordée au comportement institutionnel est certes le "plan de programme individualisé » qui apparaît de plus en plus comme la pierre angulaire de la nouvelle gestion de cas. C'est de ce plan, appelé aussi «plan de séjour» que nous discuterons maintenant.

Le plan de séjour : un carcan?

Au départ, soulignons que le plan de programme individualisé ou plan de séjour est toujours susceptible d'être modifié. En principe, donc il devrait s'agir d'un instrument souple qui permette au détenu de prendre conscience de quelques-uns des facteurs qui ont pu l'amener à commettre un délit et/ou qui peuvent l'handicaper dans son fonctionnement social et ce, en lui fixant divers objectifs à atteindre tant au niveau de la formation académique et/ou professionnelle qu'au niveau de sa famille, de la communauté, de son perfectionnement personnel, de ses loisirs et finalement tout autre niveau jugé pertinent.

Un premier élément qui peut favoriser la perception de ce plan beaucoup plus comme une entrave qu'un outil de travail est qu'il doit comporter des objectifs mesurables et quantitatifs. Or, en pratique, l'E.G.C. et par ricochet l'A.G.C.C. se trouvent donc, en termes d'évaluation de l'évolution du sujet, plus ou moins limités à l'aspect comportemental. Ainsi, un individu qui rencontre 
les objectifs «mesurables et quantifiables» qui lui sont fixés devrait, logiquement, s'attendre à une quelconque forme de libération. Or l'expérience le montre, un bon comportement institutionnel n'est pas le seul gage de réussite de la réinsertion sociale d'un sujet. De plus, baser uniquement une évaluation sur le bon comportement d'un individu favorise, à long terme, chez les concernés, la création d'une seconde nature où les problèmes de fonds sont mis en veilleuse, le temps de l'incarcération.

Un second élément qui, lui aussi, peut nous faire percevoir le plan de séjour comme un carcan est l'importance très grande qui lui est donnée au niveau du détenu et de l'E.G.C. qui doit le réévaluer régulièrement, comparativement à la valeur qui lui est accordée par les instances décisionnelles; la C.N.L.C. lors des libérations et dans certains cas d'absences temporaires qui ne relèvent pas de la Commission des libérations.

Lorsque ces instances décisionnelles sont en accord avec les étapes inscrites dans le plan de séjour, il y a peu de problèmes. Par contre, lorsqu'il y a désaccord, le premier touché est le détenu. D'une part, son séjour au pénitencier lui paraît relié à la réalisation des objectifs inscrits dans son plan de séjour et, d'autre part, ceux qui ont à prendre des décisions dans son cas ne sont pas, eux, liés par ce plan. Avec le premier refus vient alors l'impression, pour le sujet, qu'il est lié par un contrat à sens unique et avec les suivants s'installe peu à peu l'idée que, quoi qu'il fasse, la décision relative à l'amorce d'un processus de remise en liberté ne sera pas influencée.

Sans dénigrer la philosophie à la base du plan de séjour qui est de permettre une plus grande cohérence au niveau de la gestion de la sentence, de faire participer le détenu à cette gestion en établissant, avec lui, ses besoins et en fixant des objectifs à atteindre, nous ne pouvons que constater que l'écart avec la réalité est très grand.

Pour ne reprendre que quelques-uns des problèmes vécus le plus couramment dans l'établissement des plans de séjour notons : le choix du degré de sécurité dans lequel est dirigé un sujet $v s$ les institutions offrant la formation désirée par le sujet, et/ou la formation désirée $v s$ les disponibilités de l'institution (quota par atelier), la difficulté de définir, en fonction des besoins de l'individu, des objectifs " mesurables», les besoins de l'individu que ce soit au niveau personnel et/ou professionnel et 
ses problèmes dans la population carcérale (cas de protection), finalement les besoins de l'individu aux niveaux personnel et/ou professionnel et les dates d'admissibilité aux diverses formes de libération (sentences-vie).

Ce sont ces divers problèmes, auxquels il est confronté quotidiennement, qui peuvent amener l'agent de gestion de cas à percevoir le plan de séjour comme un carcan. Au fil du temps une adaptation de la part des personnes (A.G.C.E., A.U.R., A.G.C.C., psychologue, etc.) et des instances impliquées (institutions, C.N.L.C.) devra nécessairement se faire. Si cette adaptation ne se réalise pas, si les ressources nécessaires pour répondre aux besoins des individus ne sont pas élargies et révisées, si les instances décisionnelles ne s'entendent pas pour accorder la même importance au plan de séjour que celle demandée par l'A.G.C.C. et par l'E.G.C., alors il y a fort à craindre un désengagement de plus en plus grand de la part du personnel impliqué et du détenu lui-même qui ne verra d'ailleurs aucun intérêt à participer à un plan de séjour.

\section{Problèmes éthiques soulevés par la nouvelle gestion de cas}

Nous l'avons vu, la nouvelle gestion de cas, peut, au seul niveau de l'établissement du plan de séjour, mettre l'A.G.C.C. dans une situation ambiguë : d'une part, il doit participer à l'établissement de ce plan et par le fait même, montrer au détenu la nécessité tout comme, dans un rapport soumis en vue d'une libération, il doit souligner où en est le sujet dans son plan de séjour (comportement institutionnel) alors que, d'autre part, les instances décisionnelles ne sont pas, elles, liées par la réalisation des objectifs contenus par le sujet dans son plan de séjour.

La nouvelle gestion de cas peut, aussi, amener l'A.G.C.C. à vivre des situations ambiguës à d'autres niveaux et même lui poser des problèmes d'ordre éthique.

La «Loi canadienne sur les droits de la personne » énonce le principe que "chaque individu a le droit de prendre normalement connaissance du contenu d'un dossier fait à son sujet » (art. 50 al. 2b). Par ailleurs, quant aux renseignements fournis au demandeur, la loi est explicite en ce qui concerne les individus ayant été condamnés pour une infraction à une loi du Parlement (art. 54 al. 1d). C'est d'ailleurs en fonction de cet article que lors de la remise d'un dossier à un détenu ou ex-détenu, il y avait, 
presque toujours, des dispenses accordées aux rapports fournis par l'agent de libération, à certaines évaluations communautaires, au rapport de police, etc.

Avec la mise en application de la nouvelle gestion de cas, la situation a changé puisque le rapport qui sera soumis à la C.N.L.C. est contresigné par le détenu après qu'il ait pris connaissance de son contenu.

Au départ, ce qu'il faut bien comprendre c'est que lorsqu'une E.G.C. évalue un sujet, elle tire ses informations de plusieurs sources (rapport de police, évaluations communautaires, enquêtes de la sécurité préventive, rapports psychologique et/ou psychiatriques lorsqu'il y a lieu, et tout autre renseignement fourni qui lui paraît nécessaire à l'analyse du cas). Donc, elle ne peut pas se limiter à l'attitude du sujet (réalisation du plan de séjour) pour formuler une recommandation en vue d'une libération, et ce, même si une bonne partie du rapport qui sera soumis à la C.N.L.C. doit porter sur le comportement institutionnel.

Or, ces divers renseignements peuvent influencer fortement une recommandation. Ces informations sont, par contre assez régulièrement dites sous le sceau du secret et il est du ressort du professionnel de préserver cette confidentialité. Il lui est permis de joindre au rapport présenté au sujet du détenu un second rapport, confidentiel cette fois. Toutefois, si les informations confidentielles motivent en bonne partie une recommandation défavorable, surtout dans les cas où en institution le comportement du sujet est adéquat et qu'il respecte son plan de séjour, il devient malaisé de justifier auprès du détenu, une recommandation négative sans trahir la confidentialité des informations.

Dans ces cas, moins exceptionnels que l'on pourrait croire, l'agent de gestion est placé dans une situation particulièrement ambiguë. Il est difficile d'établir une ligne de conduite qui permette à la fois de préserver le caractère confidentiel des informations et le droit du concerné à connaître le contenu du rapport écrit à son sujet. D'une manière générale ce sont les membres de l'E.G.C. qui perdront la confiance du sujet puisqu'ils favoriseront la confidentialité de l'information. Le détenu, quant à lui, ne pourra que se poser une multitude de questions car il saura qu'il y a « anguille sous roche». Comment alors l'E.G.C. pourra-t-elle justifier auprès du prisonnier la nécessité de modifier ou d'établir un nouveau plan de séjour? 
En fait, ces problèmes d'éthique qui se posent à l'A.G.C.C. et aussi aux divers membres de l'E.G.C., pourront, à la longue, diminuer la qualité de l'intervention faite avec le sujet puisque ce dernier ne pourra percevoir cette E.G.C. que comme ne jouant pas « franc jeu » avec lui.

Quelle orientation l'A.G.C.C. doit-il prendre? L'agent de gestion de cas affronte un dilemme : d'une part il est intéressé à préserver la qualité de ses interventions donc de l'évaluation faite dans chacun des cas dans lequel il est appelé à travailler, d'autre part, la nouvelle gestion de cas le confronte à divers problèmes auxquels il doit quotidiennement faire face et qui limitent, souvent, la qualité de son intervention.

En outre, la nouvelle gestion de cas lui demande une présence, à l'institution, beaucoup plus grande, de même il est appelé à rencontrer un plus grand nombre de détenus en assistant à divers comités (établissement du P.P.I., participation à la première étude pour absence temporaire, assistance au comité pour un transfert, présence bi-annuelle à l'étude sur l'évolution du cas et bien entendu, sa présence est aussi requise pour les études en vue de libérations). Il lui reste, finalement, beaucoup moins de temps à consacrer à chaque étude et c'est peut-être en termes de qualité que ce manque de temps pourra le plus se faire sentir.

L'A.G.C.C. devra, durant les années à venir, se réorienter. De même, le Service correctionnel sera amené, lui aussi, à prévoir des solutions aux problèmes posés par l'implantation de la nouvelle gestion de cas. C'est par divers ajustements que finalement la gestion de cas pourra peut-être réellement atteindre les buts pour lesquels elle a été implantée. Pour ce faire, il faudra, nécessairement, favoriser la qualité de l'intervention et celle de l'évaluation plutôt que d'insister sur une rentabilisation exagérée qui ne pourrait être obtenue que par une diminution des services offerts par l'A.G.C.C. chargé de la préparation de cas.

La question essentielle toutefois demeure : l'application de la nouvelle gestion de cas est-elle, en fait, le premier pas qui mènera à la disparition de la préparation de cas? Cette question nous ne pouvons nous empêcher de nous la poser en relevant les liens étroits qui existent entre la gestion de cas et le modèle américain de la sentence déterminée. 


\section{LA TENDANCE AMÉRICAINE ET LA NOUVELLE GESTION DE CAS}

Doit-on voir dans la nouvelle gestion de cas un mouvement vers Ia tendance américaine où la sentence déterminée et sa philosophie sous-jacente prennent de l'ampleur? On peut, de toute manière faire certains rapprochements entre l'orientation actuelle du Service correctionnel canadien et l'orientation américaine.

\section{La sentence déterminée et ses fondements}

Durant plusieurs années, les sciences humaines ont joué un rôle important dans le domaine de la délinquance. Aux Etats-Unis comme au Canada, on mettait l'accent sur les notions d'individualité et de réhabilitation dans l'imposition des sentences et dans l'administration de celles-ci.

Avec la sentence indéterminée, un même délit pouvait mériter à son auteur toute une gamme de peines compte tenu de la nature du délit mais aussi de l'individu. Le prononcé de la sentence se faisait en considérant une série de facteurs particuliers au délinquant. On misait aussi sur les possibilités de changement de l'individu grâce à l'intervention des sciences humaines.

De plus en plus, cependant, les principes de la sentence indéterminée sont ébranlés, aux États-Unis surtout où la notion d'individualité a entraîné de nombreuses critiques, particulièrement de la part des détenus.

En effet, la sentence indéterminée entraîne beaucoup de disparités et ce, à un point tel que dans certains états américains, la libération conditionnelle a pour but de les corriger.

Un autre facteur ayant remis en question les valeurs sousjacentes de la sentence indéterminée réside dans le fait qu'après avoir tenté plusieurs expériences et types d'intervention mettant tantôt l'accent sur la confiance, tantôt sur la relation, tantôt sur la relation et le contrôle, les spécialistes des sciences humaines ne semblent pas avoir prouvé leur efficacité à enrayer la délinquance donnant ainsi prise aux critiques de la réhabilitation.

Le résultat concret de ces mouvements est la disparition de la notion de réhabilitation qui fait place aux notions de punition et de protection de la société. De plus, l'individualité du délinquant perd son importance.

L'État de Californie offre l'exemple le plus poussé de l'application de la sentence déterminée. Dans ce système, la sentence est imposée en fonction du délit et non de l'individu. En Californie, la libération conditionnelle n'existe pratiquement plus. 
Le Service correctionnel canadien dans son orientation actuelle s'inspire fortement de la tendance américaine. Les objectifs du S.C.C. consistent désormais à administrer la sentence, incluant le pouvoir d'en modifier la qualité selon les lois en vigueur; de protéger la société et le délinquant; de protéger les droits des délinquants et, enfin, de lui fournir des occasions de participer à des programmes susceptibles de l'aider à effectuer des choix plus judicieux.

À travers ces objectifs, la notion de réhabilitation a fait place à la notion de responsabilité qui constitue désormais la pierre angulaire de notre système pénitencier.

Il appartient au détenu de faire le choix de ses objectifs et des programmes en fonction de ses besoins et il appartient au S.C.C. de fournir les ressources et les stimuli nécessaires au détenu qui, ultérieurement, choisit de s'en servir ou de ne pas le faire. Le poids est du côté du détenu et le S.C.C. est libéré de la lourde responsabilité de la réhabilitation.

Certaines pratiques du nouveau système suggèrent étrangement le principe punitif de la sentence déterminée puisqu'elles se fondent sur la gravité objective de l'ensemble des délits commis par le détenu. Il y a de moins en moins de place pour une appréciation différenciée de chaque cas. En effet, dès le départ, le programme de certains détenus sera balisé par des critères établis à l'avance qui détermineront le placement pénitencier. Ainsi, le niveau sécuritaire de l'institution où ils seront dirigés sera établi en fonction de critères clairement explicités dans le manuel de gestion de cas et considérant les faits : nature du délit, conduite antérieure (évasion, comportements agressifs, troubles de personnalité impliquant des risques de violence). Le détenu concerné par ces critères n'aura guère d'autres choix que de débuter son « programme » en allant passer quelques années dans une institution à sécurité maximale.

Ce sont là des indices concrets qui nous amènent à penser que le Système correctionnel canadien s'oriente moins vers les possibilités de changement de l'individu que vers une organisation rationnelle du temps que celui-ci a à purger.

CONCLUSIONS

Nous l'avons vu, la nouvelle gestion de cas se définit comme un processus continu d'administration d'une sentence qui s'ap- 
plique de l'admission au pénitencier jusqu'à l'expiration de la peine.

Les conséquences logiques de cette orientation sont déjà manifestes dans l'intégration de l'agent des libérations conditionnelles à l'équipe de gestion de cas. Cette intégration détermine la disparition du processus d'évaluation "parallèle » ou indépendante de l'agent des libérations conditionnelles. Celui-ci sera désormais lié, dès le départ, par les décisions prises au pénitencier.

À plus long terme, on devrait également assister à la disparition de la Commission nationale des libérations conditionnelles. Cet organisme fera bientôt figure d'un intrus dans le processus de gestion de cas. Comme il s'agit d'un système indépendant et, qu'à date, rien n'indique que sa philosophie veuille rejoindre celle du S.C.C., les deux pouvoirs risquent, de toute évidence, de se trouver rapidement en conflit. On peut s'attendre à ce que la C.N.L.C. conserve parmi ses critères d'évaluation le redressement et la réhabilitation du détenu, contrairement au S.C.C. qui mettra plutôt l'accent sur la réalisation du plan de séjour. La cohérence si chère au S.C.C. sera dangereusement ébranlée par l'intervention de Ia C.N.L.C. et on ne se cache pas à l'administration du S.C.C. de vouloir éliminer ce pouvoir décisionnel.

Bien que le pouvoir discrétionnaire des juges et de la Commission nationale des libérations conditionnelles n'ait pas encore soulevé de mouvements vigoureux dans notre pays et que les nouvelles orientations de nos pénitenciers ne semblent pas déterminées par ce phénomène, l'application et les implications de la nouvelle gestion de cas soulèvent néanmoins la question de savoir si les critères de mise en liberté seront désormais plus « justes».

La disparition de la C.N.L.C. ne pourra, par ailleurs, qu'entraîner celle de l'agent de gestion chargé de la préparation de cas. La remise en liberté du détenu ne dépendra donc que du personnel institutionnel.

La qualité de l'évaluạtion, concept si important aux yeux de l'agent chargé de la préparation de cas, risque d'en prendre un "dur coup» et finalement, le détenu réalisera peut-être, que la « réhabilitation» valait bien la « responsabilité ». 